\title{
Association between Tumor Necrosis Factor- $\alpha$ Promoter -308 G/A Polymorphism and Early Onset Sepsis in Preterm Infants
}

\author{
Tatjana Varljen, ${ }^{1}$ Olgica Rakic, ${ }^{2}$ Gordana Sekulovic, ${ }^{2}$ Biljana Jekic, ${ }^{3}$ \\ Nela Maksimovic, ${ }^{3}$ Milica Rankovic Janevski, ${ }^{2}$ Ivana Novakovic ${ }^{3}$ and \\ Tatjana Damnjanovic ${ }^{3}$ \\ ${ }^{1}$ Institute of Legal Medicine, Faculty of Medicine, University of Belgrade, Belgrade, Serbia \\ ${ }^{2}$ Institute of Neonatology, Belgrade, Serbia \\ ${ }^{3}$ Institute of Human Genetics, Faculty of Medicine, University of Belgrade, Belgrade, Serbia
}

\begin{abstract}
Early-onset neonatal sepsis (EOS) is diagnosed during the first 7 days of neonatal life and is the major cause of morbidity and mortality among preterm infants. Genetic predisposition may have an impact on EOS susceptibility and outcome. The aim of our study was to explore the association between TNF- $\alpha-308$ G/A or IL-6 -174 G/C gene polymorphism and the susceptibility and outcome of EOS in preterm infants. The study included 471 preterm infants: 282 with EOS (151 with culture proven sepsis and 131 with clinical sepsis) and 189 without infection (control group). TNF- $\alpha-308 \mathrm{G} / \mathrm{A}$ and $\mathrm{IL}-6-174 \mathrm{G} / \mathrm{C}$ were genotyped using Real-time RCR method. We observed significantly higher frequency of A allele of TNF- $\alpha-308 \mathrm{G} / \mathrm{A}$ polymorphism in blood culture proven EOS $(p=0.017)$ or clinical EOS $(p=0.025)$ compared with the control group. Logistic regression confirmed significant association between TNF- $\alpha-308 \mathrm{GA}+A A$ genotypes and development of culture proven EOS $(B=-0.718, p=0.013)$ or clinical $E O S(B=-0.602, p$ $=0.027$ ). No significant differences in IL6 -174G/C alleles or genotypes distribution have been observed between culture proven EOS group, clinical EOS group and the control group. An association between $T N F-\alpha-308$ G/A or IL-6 -174 G/C genotypes and EOS lethal outcome was not observed $(p=0.652$ and $p=$ 0.384 , respectively). According to our analysis of large cohort of preterm infants with clearly defined EOS groups, the TNF- $\alpha-308 \mathrm{~A}$ allele may be a risk factor for the EOS occurrence.
\end{abstract}

Keywords: early onset sepsis; gene polymorphism; interleukin 6; preterm infants; tumor necrosis factor alpha Tohoku J. Exp. Med., 2019 April, 247 (4), 259-264. (C) 2019 Tohoku University Medical Press

\section{Introduction}

Neonatal sepsis is caused by invasion of microbial pathogens on the normally sterile tissue of infants and the consequent neonatal inflammatory response (Lever and Mackenzie 2007; Shane et al. 2017). The incidence of sepsis in neonates with appropriate birth weight is $0.1 \%$ but it increases to up to $50 \%$ as a birth weight and gestational age decrease (Rønnestad et al. 2005a, b; Shane and Stoll 2014). Despite a significant improvement in quality of intensive neonatal care, neonatal sepsis is the major cause of morbidity and mortality among preterm infants.

Early onset sepsis (EOS) is diagnosed during the first 7 days of neonatal life and is caused by maternal-fetal pathogens transmission through placenta or through ascending vaginal route (Mukhopadhyay and Puopolo 2012). Besides maternal factors, virulence of infecting organism and neonatal host predisposition are also recognized as risk factors for neonatal sepsis (Mukhopadhyay and Puopolo
2012).

Numerous studies have provided evidence that a genetic base may have an impact on host immunity and susceptibility and outcome of sepsis (Bellamy and Hill 1998; Cooke and Hill 2001; Waterer and Wunderink 2003; Sutherland and Walley 2009). Preterm infants have unbalanced immune response to pathogens because of the immaturity of immune system. Inflammatory response to bacterial sepsis of a neonate is mediated by cytokines through a cascade that starts with the activation of macrophages. Activated macrophages then release tumor necrosis factor alpha (TNF- $\alpha$ ) and interleukin 6 (Weirich et al. 1998; Levy et al. 2006).

TNF- $\alpha$, which is an initiator of the inflammatory response, increases in the early phase of neonatal sepsis (Meem et al. 2011). The most extensively studied polymorphism in the $T N F-\alpha$ gene is located 308 bp upstream of the transcriptional start site (rs1800629). Presence of TNF- $\alpha$ $-308 \mathrm{~A}$ allele is associated with enhanced $T N F-\alpha$ transcrip-

Received January 17, 2019; revised and accepted April 8, 2019. Published online April 19, 2019; doi: 10.1620/tjem.247.259.

Correspondence: Tatjana Damnjanovic, M.D., Ph.D., Institute of Human Genetics, Faculty of Medicine, University of Belgrade, 26

Visegradska Str., 11000 Belgrade, Serbia.

e-mail: tatjana.damnjanovic@med.bg.ac.rs 
tion (Kroeger et al. 1997) and higher production of TNF- $\alpha$ (Louis et al. 1998) in adults, but there are opposing data in neonatal patients (Allam et al. 2015). Recent data provide inconsistent results about the role of TNF -308 G/A polymorphism in neonatal sepsis. Namely, there are results that support association $T N F-\alpha-308 G$ allele with EOS in neonates (Allam et al. 2015), but there are studies that deny association between this polymorphism and culture proven neonatal sepsis (Härtel et al. 2011; Srinivasan et al. 2017).

Interleukin 6 (IL-6) plays an important role in the acute phase response to microbial invasion (Borden and Chin 1994), in the systemic inflammation, in the pathogenesis of sepsis and is associated with increased severe sepsis risk and mortality (Uusitalo-Seppälä et al. 2011; Tschaikowsky et al. 2011; Miguel-Bayarri et al. 2012; Palmiere and Augsburger 2014). The polymorphism -174 $G / C$ resides upstream of the transcriptional start site, affects transcriptional activity of the $I L-6$ gene and is associated with elevated IL-6 level in stimulated neonatal cells, but not in adult ones (Kilpinen et al. 2001).

Analysis of the polymorphisms in genes for proinflammatory cytokines $T N F-\alpha$ and $I L-6$ in neonatal sepsis may allow for the development of new diagnostic markers and improve diagnosis of sepsis, and reveal predictors of patient outcomes. This is extremely important in premature neonates who are at the highest risk of developing severe bacterial infections (Abu-Maziad et al. 2010). Inconclusive results for premature infants from previous studies may be due to differences in study design, ethnical origin and small cohort size. Our study included homogenous, large cohort with clearly defined EOS groups.

The aim of our study was to evaluate the relationships between susceptibility and outcome of early onset sepsis and $T N F-\alpha-308 \mathrm{G} / \mathrm{A}$ or $I L-6-174 \mathrm{G} / \mathrm{C}$ gene polymorphism in Serbian preterm infants.

\section{Materials and Methods}

\section{Study population}

Our study enrolled 471 preterm infants (gestational age $<37$ weeks) who were admitted to the Institute of Neonatology in Belgrade, Serbia, from January 2012 to September 2018. The study protocol was approved by the Ethics Committee Institute of the Neonatology in Belgrade and the Ethics Committee of the Faculty of Medicine, University of Belgrade. In all cases, the infants' parents were informed and their written consent was obtained.

\section{Study design}

Neonates with sepsis are diagnosed during the first 7 days of neonatal life and classified as EOS. Premature neonates with EOS (n $=282$ ) were divided into the group with blood culture proven sepsis $(\mathrm{n}=151)$ and the group with symptoms of clinical sepsis but negative blood culture $(n=131)$. The control group consisted of 189 preterm neonates with negative blood culture and with no symptoms of clinical sepsis during hospitalization.

Clinical sepsis was defined as the presence of at least three out of six clinical and three out of nine laboratory criteria, according to the protocol of the Institute of Neonatology (http://www.neona
tologija.rs/files/rad16-KON-2.pdf) based on modified Töllner and Rodwell criteria (Töllner 1982; Rodwell et al. 1988). The clinical symptoms of sepsis are 1) apnea, dyspnea, tachypnea, cyanosis; 2) tachycardia, hypotension, bradycardia; 3) lethargy, hypotonia, irritability, convulsions; 4) poor perfusion, arterial hypotension; 5) feeding intolerance or abdominal distension; and 6) hepatosplenomegaly, jaundice or skin and subcutaneous lesions.

The laboratory criteria for detecting sepsis according to the hematological scoring system (HSS) of Rodwell et al. (1988) are: 1) a total white blood cell (WBC) count $\leq 5,000 / \mu 1$ or $\geq 25,000 / \mu 1 ; 2$ ) a total polymorphonuclear leukocytes $(\mathrm{PMN})$ count $<1,800 / \mu 1$ or $>5,400 / \mu 1$, or no mature PMN seen; 3 ) increased immature PMN count from normal value $600 / \mu 1 ; 4)$ an immature to total neutrophil ratio $(\mathrm{I} / \mathrm{T})$ of $\geq 0.2 ; 5)$ an immature to mature neutrophil ratio $(\mathrm{I} / \mathrm{M})$ of $\geq 0.3$; 6) presence of degenerative morphologic changes in PMN; 7) a platelet count $\leq 150,000 / \mu 1 ; 8)$ C-reactive protein (CRP) levels greater than $5 \mathrm{mg} / \mathrm{L}$; and 9) acidosis as characterized by a base excess (BE) of $0.210 \mathrm{mmol} / \mathrm{L}$.

For all neonates, the data about gender, gestational age, weight on birth (BMW), Apgar score determined in the 5th minute (AS5'), type of delivery and multiple pregnancies were collected.

Possible occurrence of intracranial hemorrhage (ICH) in patients was analyzed by ultrasound of the central nervous system (Papile et al. 1978). Respiratory distress syndrome (RDS) was confirmed by assessment of Chest X-rays (Bomsel 1970).

\section{Sampling and analyses}

Genomic DNA from buccal swabs taken from patients was extracted using the conventional phenol: chloroform-based method. All molecular genetic analyses were conducted at the Institute of Human Genetics, Faculty of Medicine University of Belgrade.

In our study, we analyzed the single nucleotide polymorphisms of TNF- $\alpha(-308 \mathrm{G} / \mathrm{A}, \mathrm{rs} 1800629)$ and $I L-6$ (-174 G/C, rs1800795) genes. Genotyping was performed with custom TaqMan ${ }^{\circledR}$ SNP Genotyping Assays and TaqMan ${ }^{\circledR}$ Genotyping Master Mix (Applied Biosystems, Foster City, CA) in $10 \mu \mathrm{L}$ volumes. Genotype analyses were carried out in Applied Biosystems' 7500 Real-Time PCR System (Applied Biosystems).

\section{Statistical analysis}

Mean values between groups of neonatal patients were compared by ANOVA or Kruskal-Wallis test, depending on variable distribution. Differences in genotype and allele frequencies between the group with blood culture proven sepsis, the one with clinical sepsis and the control group were analyzed by Chi-square test and Fisher exact test. Association between analyzed polymorphisms and sepsis occurrence or outcome in preterm infants with sepsis was explored by logistic regression analysis after the adjustment for BMW, gestational age, Apgar score and culture proven sepsis. Statistical analyses were performed using SPSS statistical package, version 16.0 (SPSS Inc, Chicago, IL, USA).

\section{Results}

We analyzed 471 premature infants. EOS groups included 151 (32.1\%) preterm infants with culture proven sepsis and $131(27.8 \%)$ with clinical sepsis. The control group included 189 (40.1\%) preterm infants who had no signs of sepsis during hospitalization and did not require any antimicrobial treatment. The clinical data of the ana- 
lyzed infants are shown in Table 1.

Gestational age, weight mass at birth and Apgar score were statistically significantly lower in the culture proven EOS group and the clinical EOS group than in the control group. In the culture proven EOS group and the clinical EOS group frequencies of intracranial hemorrhage and death were statistically significantly higher, while caesarean section delivery was significantly lower than in the control group (Table 1).

In preterm infants with positive blood culture, 106 (70.2\%) cases of sepsis were caused by Gram-negative bacteria and 45 (29.8\%) by Gram-positive bacteria.

Both analyzed polymorphisms in premature infants were in Hardy-Weinberg equilibrium (Table 2). We observed statistically significant differences in $T N F-\alpha$ $-308 \mathrm{G} / \mathrm{A}$ polymorphism alleles frequencies between blood culture proven EOS $(p=0.017)$ or clinical EOS $(p=0.025)$ and the control group of premature infants (Table 2). Also, a statistically significant difference was observed in $T N F-\alpha$ $-308 G / A$ genotypes distribution between the culture proven EOS group and the control group by dominant model (GG vs. GA/AA) ( $\mathrm{p}=0.011$; Risk ratio $1.40,95 \%$ CI 1.047 1.869; Odds ratio $1.98,95 \%$ CI 1.163-3.356). A similar trend was observed between the clinical EOS group and the control group by dominant model $(\mathrm{p}=0.057$; Risk ratio $1.27,95 \%$ CI $0.968-1.676$; Odds ratio $1.71,95 \%$ CI 0.981 2.992). The frequency of A allele $T N F-\alpha-308 G / A$ polymorphism is significantly higher in the culture proven EOS group and the clinical EOS group than in the control group (Table 2). After the adjustment for BMW, gestational age, AS5' and type of delivery, logistic regression confirmed statistically significant association between $T N F-\alpha-308 G / A$ genotypes and development of culture proven EOS $(\mathrm{B}=$ $-0.718, p=0.013)$ or clinical EOS $(B=-0.602, p=0.027)$ in premature infants.

Genotype and allele frequencies of $I L-6$ gene $-174 G / C$ polymorphism among the culture proven EOS group, the clinical EOS group and the control group did not show significant differences (Table 2). Also, genotype frequencies among the culture proven EOS group, the clinical EOS group and the control group by recessive (GG/GC vs. CC) model did not show significant differences.

$T N F-\alpha-308 G / A$ genotypes distribution in infants who $\mathrm{did} / \mathrm{did}$ not die because of sepsis were: AA $1.2 \%$, GA $23.5 \%$, GG $75.3 \%$ and AA $2.5 \%$, GA $25.2 \%$, GG $72.3 \%$ respectively $(\mathrm{p}=0.652)$. $I L-6-174 G / C$ genotypes distribution in infants who did/did not die because of sepsis were: $\mathrm{CC} 10.8 \%$, GC $42.8 \%$, GG $46.4 \%$ and $\mathrm{CC} 14.3 \%$, GC $47.1 \%$, GG $38.7 \%$ respectively $(\mathrm{p}=0.384)$. In the EOS group $T N F-\alpha-308 G / A$ and $I L-6-174 G / C$ genotypes were not predictors of lethal EOS outcome.

\section{Discussion}

Pathophysiology of sepsis is a complex process which includes interaction of microorganisms with the innate and adaptive host immune system. Fragile immature skin and immature mucosal barriers as well as delayed maturation of the specific humoral and cellular immune response and complete physiological immaturity are some of the key reasons why sepsis is the main cause of morbidity and mortality of preterm infants (Kamalakannan 2018). The most relevant risk factors associated with EOS are low gestational age and low birth weight and Apgar Score $\leq 6$ at $5 \mathrm{~min}$ (Simonsen et al. 2014). Our study confirmed these risk factors for development of EOS. As expected, vaginal delivery is significantly more frequent in our septic group infants because of the exposure of infants to microorganisms in birth canal (Plano 2010). Gram-negative bacteria are the

Table 1. Comparison of clinical data of preterm neonates with and without sepsis.

\begin{tabular}{|c|c|c|c|c|}
\hline Clinical characteristics & Culture proven EOS & Clinical EOS & Control group & $\mathrm{p}$ values \\
\hline GA weeks" & $27.8 \pm 3.2$ & $28.5 \pm 3.4$ & $30.2 \pm 3.4$ & 0.000 \\
\hline BWM grams* & $1,018.8 \pm 410.4$ & $1,161.8 \pm 510.3$ & $1,363.7 \pm 460.0$ & 0.000 \\
\hline Male & $85(56.3)$ & $74(56.5)$ & $97(51.3)$ & $>0.5$ \\
\hline AS 5,* & $3.7 \pm 2.3$ & $3.9 \pm 2.3$ & $5.4 \pm 2.4$ & 0.000 \\
\hline C-section delivery ${ }^{* *}$ & $66(43.7)$ & $57(43.5)$ & $113(59.8)$ & 0.003 \\
\hline Multiple births $^{* *}$ & $39(25.8)$ & $36(27.5)$ & 64 (33.9) & $>0.5$ \\
\hline $\operatorname{RDS}^{* *}$ & $145(96.0)$ & $122(93.1)$ & $176(93.1)$ & $>0.5$ \\
\hline $\mathrm{ICH}^{* *}$ & $30(19.9)$ & $30(22.9)$ & $16(8.5)$ & 0.001 \\
\hline Death $^{* *}$ & 98 (64.9) & $67(51.5)$ & 63 (33.7) & 0.000 \\
\hline
\end{tabular}

$*$ mean value $\pm \mathrm{SD} ; * *$ No $(\%)$.

GA, gestational age; BWM, weight on birth; AS 5', apgar score at 5th minute; RDS, respiratory distress syndrome; $\mathrm{ICH}$, intracranial hemorrhage. 
Table 2. Analysis of genotypes and alleles distribution of $T N F-\alpha-308 G / A$ and $I L-6$ -174 polymorphisms in the study group.

\begin{tabular}{|c|c|c|c|c|c|}
\hline Polymorphism & Genotype & $\begin{array}{c}\text { Culture proven } \\
\text { EOS n }(\%)\end{array}$ & $\begin{array}{c}\text { Clinical EOS } \\
\text { n }(\%)\end{array}$ & $\begin{array}{c}\text { Control group } \\
\text { n (\%) }\end{array}$ & ${ }^{*}$ p value \\
\hline \multirow{3}{*}{$T N F-\alpha-308 G / A$} & GG & $110(72.8)$ & $99(75.6)$ & $159(84.1)$ & \multirow{3}{*}{0.010} \\
\hline & GA & $40(26.5)$ & $28(21.4)$ & $30(15.9)$ & \\
\hline & AA & $1(0.8)$ & $4(3.0)$ & $0(0)$ & \\
\hline \multirow{2}{*}{$T N F-\alpha-308 G / A$} & GG & $110(72.8)$ & $99(75.6)$ & $159(84.1)$ & \multirow[b]{2}{*}{0.031} \\
\hline & $\mathrm{GA}+\mathrm{AA}$ & $41(27.2)$ & $32(24.4)$ & $30(15.9)$ & \\
\hline \multirow{2}{*}{ Allele \% } & G & 86.1 & 86.3 & 92.1 & \multirow[b]{2}{*}{0.035} \\
\hline & A & 13.9 & 13.7 & 7.9 & \\
\hline \multirow{3}{*}{$I L-6-174 G / C$} & GG & $65(43.0)$ & $57(43.5)$ & $84(44.4)$ & \multirow{3}{*}{0.878} \\
\hline & GC & $66(43.7)$ & $61(46.6)$ & $80(42.3)$ & \\
\hline & $\mathrm{CC}$ & $20(13.2)$ & $13(9.9)$ & $25(13.2)$ & \\
\hline \multirow{2}{*}{$I L-6-174 G / C$} & $\mathrm{GG}+\mathrm{GC}$ & $131(86.8)$ & $118(90.1)$ & $164(86.8)$ & \multirow{2}{*}{0.619} \\
\hline & $\mathrm{CC}$ & $20(13.2)$ & $13(9.9)$ & $25(13.2)$ & \\
\hline \multirow{2}{*}{ Allele \% } & $\mathrm{G}$ & 64.9 & 66.8 & 65.6 & \multirow{2}{*}{0.887} \\
\hline & $\mathrm{C}$ & 35.1 & 33.2 & 34.4 & \\
\hline
\end{tabular}

*Chi-square test or Fisher exact test.

most commonly isolated pathogens in positive blood cultures of our septic infants, and those findings are in concordance with previous studies (Plano 2010; Cortese et al. 2016).

TNF- $\alpha$ and IL- 6 are primary proinflammatory cytokines that mediate the initial response of the innate immune system to infection and have an important role in the pathogenesis of sepsis. Previous studies have shown that septic neonates had elevated levels of circulating TNF- $\alpha$ and IL-6 cytokines in plasma when compared with healthy infants (Kurt et al. 2007; Allam et al. 2015). These cytokines were classified as biomarkers of early phase of EOS (Meem et al. 2011).

TNF- $\alpha$ plays a prominent role in the acute inflammatory response through induction of release of numerous inflammatory mediators and through activation of immune and structural cells (El-Tahan et al. 2016). Genetic variations in $T N F-\alpha$ gene may affect circulatory levels of TNF- $\alpha$ (Sutherland and Walley 2009; Wynn and Wong 2010). Allele A in $T N F-\alpha-308$ G/A polymorphism is associated with higher expression of $T N F-\alpha$ and increased susceptibility to sepsis and septic shock in adults (Mira et al. 1999). Our study showed association between $T N F-\alpha-308 \mathrm{~A}$ allele and the risk of culture proven and clinical EOS in preterm infants. Moreover, all analyzed preterm infants with $T N F-\alpha$
$A A$ genotype developed EOS. Previous studies have shown that the AA genotype has affected TNF- $\alpha$ production, secretion, or function (El-Tahan et al. 2016). Abnormal TNF- $\alpha$ is implicated in the pathogenesis of sepsis (Zhang et al. 2017), and TNF- $\alpha$ gene expression is closely related to neonatal sepsis in very low birth weight infants (Cernada et al. 2014). In our study a significant association was found between TNF- $\alpha-308$ G/A polymorphism and EOS (culture proven and clinical) under the dominant model (for GG vs. GA/AA). A strong association between the $T N F-\alpha-308 \mathrm{G} /$ $A$ polymorphism and EOS in premature infants is confirmed by logistic regression (covariates BMW, GA, AS5' and type of delivery).

A recently published meta-analysis with pooled data from 34 publications that included 12,284 subjects (only 4 studies included neonates) suggested that $T N F-\alpha-308 \mathrm{G} / \mathrm{A}$ polymorphism may contribute to risk of sepsis and septic shock under the dominant model (Wang et al. 2017). The results are consistent in part with the present study. Moreover, to the best of our knowledge, we are able to show for the first time that $T N F-\alpha-308 \mathrm{~A}$ allele is a risk factor for EOS in premature infants. Härtel and coworkers (2011) in two large cohorts of very low birth weight infants did not find an association between $T N F-\alpha-308$ G/A polymorphism and neonatal sepsis. Similar findings were also 
obtained by Srinivasan and coworkers (2017), but with the emphasis on the fact that GG genotype shows a trend towards an increased sepsis risk in infants with culture proven sepsis. A study from Saudi Arabia on newborn infants who were $40 \pm 2$ weeks of gestation has shown the association between GG genotype and $\mathrm{G}$ allele of $T N F-\alpha$ -308 G/A polymorphism with EOS (Allam et al. 2015). Inconclusive results for premature infants may be due to the differences in study design, ethnical origin and small cohort size, which could all be limiting factors. Hence, we have focused on a homogenous, large premature infant cohort with clearly defined EOS groups.

One of the first studies of the relationship between $T N F-\alpha-308 \mathrm{G} / A$ polymorphism and sepsis in infants found strong association of GA/AA genotypes and mortality (Hedberg et al. 2004). Our study did not confirm the connection of $T N F-\alpha-308$ G/A genotypes with EOS mortality.

IL-6 mediates early response to infection, it is a major inducer of the hepatic protein synthesis and it precedes the increasing C-reactive protein concentration. Because of this it is a good marker for EOS with a high degree of sensitivity and specificity (Machado et al. 2014). We did not find any association between genotype or allele distribution of $I L-6-174 G / C$ polymorphisms and culture proven and clinical EOS or EOS outcome. Numerous studies on pediatric and neonatal patients have found association of $I L-6$ $-174 \mathrm{G} / \mathrm{C}$ polymorphism with sepsis, septic shock or sepsis outcome (Harding et al. 2003; Ahrens et al. 2004; Baier et al. 2006; Wynn et al. 2010; Jabandziev et al. 2014). However, in two large meta-analyses, the authors failed to detect any association of $I L-6-174 \mathrm{G} / \mathrm{C}$ polymorphism and neonatal sepsis (Chauhan and McGuire 2008; Machado et al. 2014), the results of which are in agreement with our study.

To our knowledge, this is the first study that has found the association of $T N F-\alpha-308 G / A$ polymorphism and blood culture proven EOS or clinical EOS. Additionally, this is one of the largest cohorts of preterm neonatal patients analyzed for the association between $T N F-\alpha-308 \mathrm{G} / \mathrm{A}$ and $I L-6$ $-174 G / C$ polymorphisms and EOS. Our results suggest that $T N F-\alpha-308 G A$ and $A A$ genotypes are risk factors for EOS occurrence in Serbian population. TNF- $\alpha-308$ G/A and $I L-6-174 \mathrm{G} / \mathrm{C}$ polymorphisms are not genetic risk factors for lethal EOS outcome.

\section{Acknowledgments}

This work was supported by the Serbian Ministry of Education, Science and Technological development (Grant 175091).

\section{Conflict of Interest}

The authors declare no conflict of interest.

\section{References}

Abu-Maziad, A., Schaa, K., Bell, E.F., Dagle, J.M., Cooper, M., Marazita, M.L. \& Murray, J.C. (2010) Role of polymorphic variants as genetic modulators of infection in neonatal sepsis.
Pediatr. Res., 68, 323-329.

Ahrens, P., Kattner, E., Kohler, B., Hartel, C., Seidenberg, J., Segerer, H., Moller, J. \& Göpel, W. (2004) Mutations of genes involved in the innate immune system as predictors of sepsis in very low birth weight infants. Pediatr. Res., 55, 652-656.

Allam, G., Alsulaimani, A.A., Alzaharani, A.K. \& Nasr, A. (2015) Neonatal infections in Saudi Arabia: association with cytokine gene polymorphisms. Cent. Eur. J. Immunol., 40, 68-77.

Baier, R.J., Loggins, J. \& Yanamandra, K. (2006) IL-10, IL-6 and CD14 polymorphisms and sepsis outcome in ventilated very low birth weight infants. BMC Med., 4, 10 .

Bellamy, R. \& Hill, A.V. (1998) Genetic susceptibility to mycobacteria and other infectious pathogens in humans. Curr. Opin. Immunol., 10, 483-487.

Bomsel, F. (1970) Radiologic study of hyaline membrane disease: 110 cases. J. Radiol. Electrol. Med. Nucl., 51, 259-268.

Borden, E.C. \& Chin, P. (1994) Interleukin-6: a cytokine with potential diagnostic and therapeutic roles. J. Lab. Clin. Med., 123, 824-829.

Cernada, M., Serna, E., Bauerl, C., Collado, M.C., Perez-Martinez, G. \& Vento, M. (2014) Genome-wide expression profiles in very low birth weight infants with neonatal sepsis. Pediatrics, 133, e1203-1211.

Chauhan, M. \& McGuire, W. (2008) Interleukin-6 (-174C) polymorphism and the risk of sepsis in very low birth weight infants: meta-analysis. Arch. Dis. Child. Fetal Neonatal Ed., 93, F427-429.

Cooke, G.S. \& Hill, A.V. (2001) Genetics of susceptibility to human infectious disease. Nat. Rev. Genet., 2, 967-977.

Cortese, F., Scicchitano, P., Gesualdo, M., Filaninno, A., De Giorgi, E., Schettini, F., Laforgia, N. \& Ciccone, M.M. (2016) Early and late infections in newborns: where do we stand? A review. Pediatr. Neonatol., 57, 265-273.

El-Tahan, R.R., Ghoneim, A.M. \& El-Mashad, N. (2016) TNF- $\alpha$ gene polymorphisms and expression. Springerplus, 5, 1508.

Harding, D., Dhamrait, S., Millar, A., Humphries, S., Marlow, N., Whitelaw, A. \& Montgomery, H. (2003) Is interleukin-6 -174 genotype associated with the development of septicemia in preterm infants? Pediatrics, 112, 800-803.

Härtel, C., Hemmelmann, C., Faust, K., Gebauer, C., Hoehn, T., Kribs, A., Laux, R., Nikischin, W., Segerer, H., Teig, N., von der Wense, A., Wieg, C., Herting, E., Gopel, W. \& German Neonatal, N. (2011) Tumor necrosis factor- $\alpha$ promoter -308 G/A polymorphism and susceptibility to sepsis in very-lowbirth-weight infants. Crit. Care Med., 39, 1190-1195.

Hedberg, C.L., Adcock, K., Martin, J., Loggins, J., Kruger, T.E. \& Baier, R.J. (2004) Tumor necrosis factor alpha: 308 polymorphism associated with increased sepsis mortality in ventilated very low birth weight infants. Pediatr. Infect. Dis. J., 23, 424-428.

Jabandziev, P., Smerek, M., Michalek, J., Fedora, M., Kosinova, L., Hubacek, J.A. \& Michalek, J. (2014) Multiple gene-to-gene interactions in children with sepsis: a combination of five gene variants predicts outcome of life-threatening sepsis. Crit. Care, 18, R1.

Kamalakannan, S.K. (2018) Neonatal sepsis past to present. Biomed. J. Sci. Tech. Res., 3, 1-6.

Kilpinen, S., Hulkkonen, J., Wang, X.Y. \& Hurme, M. (2001) The promoter polymorphism of the interleukin- 6 gene regulates interleukin-6 production in neonates but not in adults. Eur. Cytokine Netw., 12, 62-68.

Kroeger, K.M., Carville, K.S. \& Abraham, L.J. (1997) The -308 tumor necrosis factor-alpha promoter polymorphism effects transcription. Mol. Immunol., 34, 391-399.

Kurt, A.N., Aygun, A.D., Godekmerdan, A., Kurt, A., Dogan, Y. \& Yilmaz, E. (2007) Serum IL-1beta, IL-6, IL-8, and TNF-alpha levels in early diagnosis and management of neonatal sepsis. Mediators Inflamm., 2007, 31397.

Lever, A. \& Mackenzie, I. (2007) Sepsis: definition, epidemiology, 
and diagnosis. $B M J, \mathbf{3 3 5}, 879-883$.

Levy, O., Coughlin, M., Cronstein, B.N., Roy, R.M., Desai, A. \& Wessels, M.R. (2006) The adenosine system selectively inhibits TLR-mediated TNF-alpha production in the human newborn. J. Immunol., 177, 1956-1966.

Louis, E., Franchimont, D., Piron, A., Gevaert, Y., SchaafLafontaine, N., Roland, S., Mahieu, P., Malaise, M., De Groote, D., Louis, R. \& Belaiche, J. (1998) Tumour necrosis factor (TNF) gene polymorphism influences TNF-alpha production in lipopolysaccharide (LPS)-stimulated whole blood cell culture in healthy humans. Clin. Exp. Immunol., 113, 401-406.

Machado, J.R., Soave, D.F., da Silva, M.V., de Menezes, L.B., Etchebehere, R.M., Monteiro, M.L., dos Reis, M.A., Correa, R.R. \& Celes, M.R. (2014) Neonatal sepsis and inflammatory mediators. Mediators Inflamm., 2014, 269681.

Meem, M., Modak, J.K., Mortuza, R., Morshed, M., Islam, M.S. \& Saha, S.K. (2011) Biomarkers for diagnosis of neonatal infections: a systematic analysis of their potential as a point-of-care diagnostics. J. Glob. Health, 1, 201-209.

Miguel-Bayarri, V., Casanoves-Laparra, E.B., Pallas-Beneyto, L., Sancho-Chinesta, S., Martin-Osorio, L.F., Tormo-Calandin, C. \& Bautista-Rentero, D. (2012) Prognostic value of the biomarkers procalcitonin, interleukin- 6 and C-reactive protein in severe sepsis. Med. Intensiva, 36, 556-562.

Mira, J.P., Cariou, A., Grall, F., Delclaux, C., Losser, M.R., Heshmati, F., Cheval, C., Monchi, M., Teboul, J.L., Riche, F., Leleu, G., Arbibe, L., Mignon, A., Delpech, M. \& Dhainaut, J.F. (1999) Association of TNF2, a TNF-alpha promoter polymorphism, with septic shock susceptibility and mortality: a multicenter study. JAMA, 282, 561-568.

Mukhopadhyay, S. \& Puopolo, K.M. (2012) Risk assessment in neonatal early onset sepsis. Semin. Perinatol., 36, 408-415.

Palmiere, C. \& Augsburger, M. (2014) Markers for sepsis diagnosis in the forensic setting: state of the art. Croat. Med. J., 55, 103-114.

Papile, L.A., Burstein, J., Burstein, R. \& Koffler, H. (1978) Incidence and evolution of subependymal and intraventricular hemorrhage: a study of infants with birth weights less than 1,500 gm. J. Pediatr., 92, 529-534.

Plano, L.R. (2010) The changing spectrum of neonatal infectious disease. J. Perinatol., 30 Suppl, S16-20.

Rodwell, R.L., Leslie, A.L. \& Tudehope, D.I. (1988) Early diagnosis of neonatal sepsis using a hematologic scoring system. J. Pediatr., 112, 761-767.

Rønnestad, A., Abrahamsen, T.G., Medbo, S., Reigstad, H., Lossius, K., Kaaresen, P.I., Egeland, T., Engelund, I.E., Irgens, L.M. \& Markestad, T. (2005a) Late-onset septicemia in a Norwegian national cohort of extremely premature infants receiving very early full human milk feeding. Pediatrics, $\mathbf{1 1 5}$, e269-276.
Rønnestad, A., Abrahamsen, T.G., Medbo, S., Reigstad, H., Lossius, K., Kaaresen, P.I., Engelund, I.E., Irgens, L.M. \& Markestad, T. (2005b) Septicemia in the first week of life in a Norwegian national cohort of extremely premature infants. Pediatrics, 115, e262-268.

Shane, A.L., Sanchez, P.J. \& Stoll, B.J. (2017) Neonatal sepsis. Lancet, 390, 1770-1780.

Shane, A.L. \& Stoll, B.J. (2014) Neonatal sepsis: progress towards improved outcomes. J. Infect., 68 Suppl 1, S24-32.

Simonsen, K.A., Anderson-Berry, A.L., Delair, S.F. \& Davies, H.D. (2014) Early-onset neonatal sepsis. Clin. Microbiol. Rev., 27, 21-47.

Srinivasan, L., Swarr, D.T., Sharma, M., Cotten, C.M. \& Kirpalani, H. (2017) Systematic review and meta-analysis: gene association studies in neonatal sepsis. Am. J. Perinatol., 34, 684-692.

Sutherland, A.M. \& Walley, K.R. (2009) Bench-to-bedside review: association of genetic variation with sepsis. Crit. Care, 13, 210.

Töllner, U. (1982) Early diagnosis of septicemia in the newborn. Clinical studies and sepsis score. Eur. J. Pediatr., 138, 331-337.

Tschaikowsky, K., Hedwig-Geissing, M., Braun, G.G. \& RadespielTroeger, M. (2011) Predictive value of procalcitonin, interleukin-6, and C-reactive protein for survival in postoperative patients with severe sepsis. J. Crit. Care, 26, 54-64.

Uusitalo-Seppälä, R., Koskinen, P., Leino, A., Peuravuori, H., Vahlberg, T. \& Rintala, E.M. (2011) Early detection of severe sepsis in the emergency room: diagnostic value of plasma C-reactive protein, procalcitonin, and interleukin-6. Scand. J. Infect. Dis., 43, 883-890.

Wang, H., Guo, S., Wan, C., Yang, T., Zeng, N., Wu, Y., Chen, L., Shen, Y. \& Wen, F. (2017) Tumor necrosis factor- $\alpha-308$ G/A polymorphism and risk of sepsis, septic shock, and mortality: an updated meta-analysis. Oncotarget, 8, 94910-94919.

Waterer, G.W. \& Wunderink, R.G. (2003) Science review: genetic variability in the systemic inflammatory response. Crit. Care, 7, 308-314.

Weirich, E., Rabin, R.L., Maldonado, Y., Benitz, W., Modler, S., Herzenberg, L.A. \& Herzenberg, L.A. (1998) Neutrophil CD11b expression as a diagnostic marker for early-onset neonatal infection. J. Pediatr., 132, 445-451.

Wynn, J., Cornell, T.T., Wong, H.R., Shanley, T.P. \& Wheeler, D.S. (2010) The host response to sepsis and developmental impact. Pediatrics, 125, 1031-1041.

Wynn, J.L. \& Wong, H.R. (2010) Pathophysiology and treatment of septic shock in neonates. Clin. Perinatol., 37, 439-479.

Zhang, Y., Cui, X., Ning, L. \& Wei, D. (2017) The effects of tumor necrosis factor- $\alpha$ (TNF- $\alpha$ ) rs1800629 and rs361525 polymorphisms on sepsis risk. Oncotarget, 8, 111456-111469. 